[RAdiocarbon, Vol. 20, No. 2, 1978, P. 171-191]

\title{
ARIZONA RADIOCARBON DATES IX: CARBON ISOTOPE DATING OF PACKRAT MIDDENS
}

\author{
JIM I MEAD,* ROBERT S THOMPSON,* and AUSTIN LONG**
}

Department of Geosciences, University of Arizona

Tucson, Arizona 85721

\section{INTRODUCTION}

The carbon isotope analyses reported here include all radiocarbon dates run on packrat middens in the United States and Mexico by the Arizona radiocarbon laboratory through October 1977. All samples described below report dates by $\mathrm{CO}_{2}(0.5$ or $2.0 \mathrm{~L})$ counting. Age calculations are based on a ${ }^{14} \mathrm{C}$ half-life of 5568 years, using 0.949 NBS oxalic acid as the modern value. Errors, based on counting statistics, are quoted to $\pm 1 \delta$; infinite ages quoted to $-2 \delta$.

Sample collectors and submitters are unless otherwise stated: Kenneth L. Cole (KLC), Austin Long (AL), Paul S Martin (PSM), Jim I Mead (JIM), W Geoffery Spaulding (WGS), Robert S Thompson (RST) and Thomas R Van Devender (TRV), Department of Geosciences, University of Arizona, Tucson; Arthur M Phillips, III (AMP), Museum of Northern Arizona, Flagstaff, Arizona; and Benjamin L Everitt (BLE), Utah Geological Survey, Salt Lake City, Utah.

Packrats (Neotoma spp) collect a variety of materials, including seeds, twigs and bones for food and constructional components in their houses and dens. These materials are collected within the home-range of the packrat, which is usually less than $100 \mathrm{~m}$ from the den (Stones and Hayward, 1968). Periodic "cleaning" of the house or den by the packrat produces a midden of discarded waste material. Repeated trampling and urination on this midden may convert it into a hard, urine-cemented (indurated) mass. The analyses of ancient packrat middens preserved in dry rock crevices or caves have provided important information about past biotic communities (Wells, 1976; Van Devender, 1977).

An indurated midden is very hard, and a hammer and chisel are usually required to remove the sample from its shelter. If the midden is stratified, its layers are separated and considered different units. In the Laboratory of Paleoenvironmental Studies, a 1 to $2 \mathrm{~kg}$ portion of each stratified midden unit is brushed to remove loose debris and placed in a $4 \mathrm{~L}$ container of water. After the cementing urine has been soaked free, the water is decanted through fine screens, leaving the midden debris for analysis. This material is then dried and the floral and faunal fossils removed by hand-picking. Generally, 5 to $10 \mathrm{~g}$ of material to be radiocarbon dated are selected from the midden unit sample, and observable extraneous material is removed. In previous years, only a cold water wash was used to remove the urine, but for the past $3 \mathrm{yr}$ an additional $30 \mathrm{~min}$ warm water wash in an ultrasonic cleaner has been used. Preparatory to com-

* Laboratory of Paleoenvironmental Studies

** Laboratory of Isotope Geochemistry 


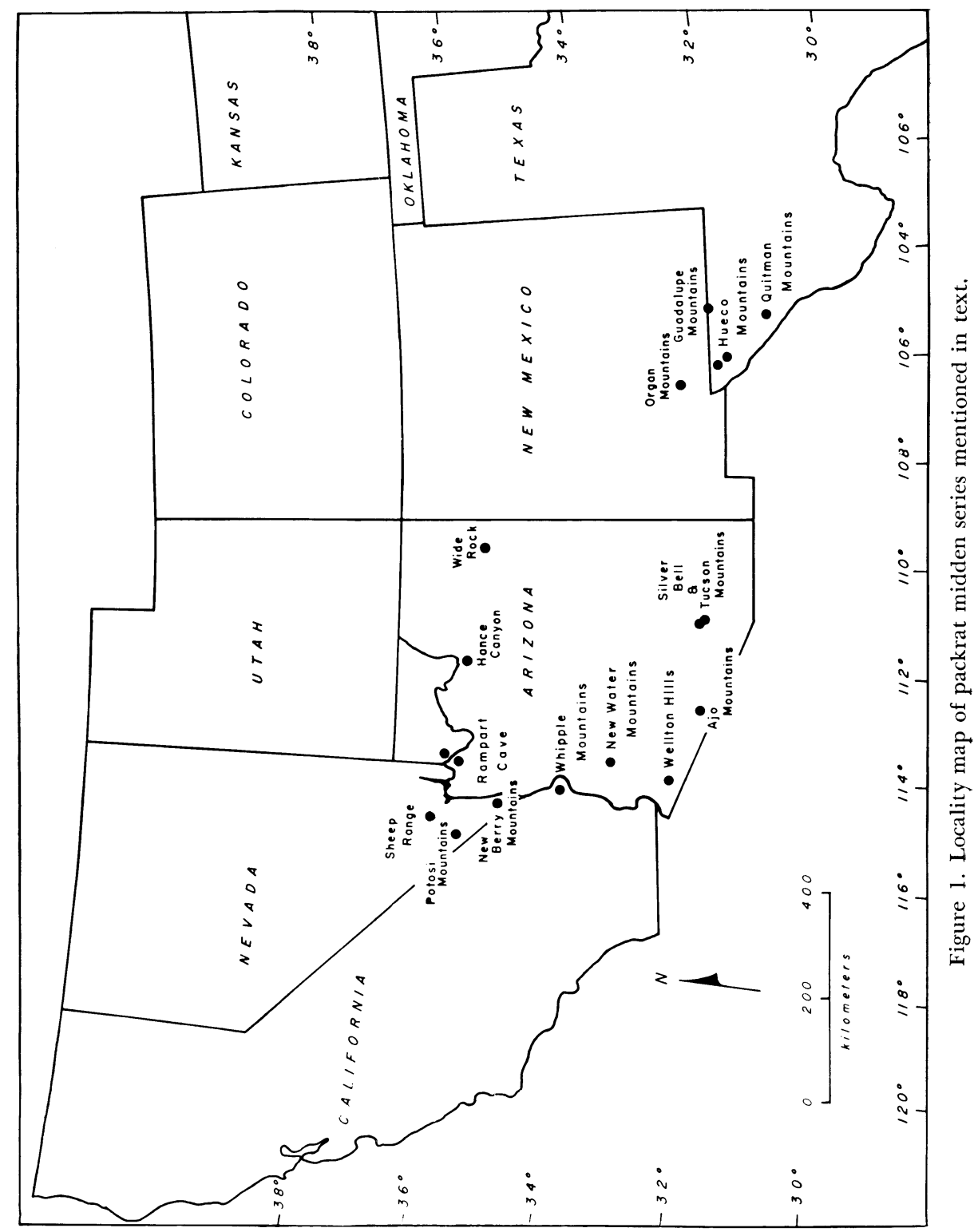


bustion in the ${ }^{14} \mathrm{C}$ laboratory, the plant fragments are treated in hot $2 \%$ $\mathrm{NaOH}$, then in hot $1 \mathrm{~N} \mathrm{HCI}$ for ca $15 \mathrm{~min}$ each. Finally the samples are rinsed in water and dried overnight.

There has been much debate concerning the best sampling procedure for radiocarbon dates from packrat middens (Wells, 1976). We believe that, in general, it is best to date a fossil species that no longer occurs in the area at present (extralocal). This method provides us with an average radiocarbon date directly on this extralocal species. A single midden unit may contain upward of 35 plant taxa. At lower alts, Juniperus usually has been the dominant taxa by weight and number, whereas, at higher alts, the dominant taxa usually are Pinus spp and Picea. Mixed in with these dominants are other less dominant extralocal and local taxa. The most abundant taxon is usually selected for radiocarbon dating because it is extralocal and because it provides the required minimum weight for a datable sample. We feel that obtaining a date on a thin bulk sample does not show direct assoc with the contents of the remaining larger portion of the midden sample. Preferably 2 radiocarbon dates should be run on each midden unit, with 1 date on an extralocal species and the other date on a local species or a bulk sample of the entire unit. Bulk midden samples may, on the one hand, represent a shorter length of time, but on the other hand, seem prone to contamination with younger urine and plant fragments. Indeed, there may be no one sampling procedure which will provide accurate dates for all middens.

The radiocarbon samples are organized by their present biogeographic or physiographic region: Colorado Plateau, Grand Canyon, Mohave Desert, Sonoran Desert or Chihuahuan Desert. Under each division, further subdivision is determined by mountain range, canyon or cave. All samples are from indurated middens unless otherwise stated.

\section{ACKNOWLEDGMENTS}

We thank Tom Van Devender, Paul Martin, Geoff Spaulding, Ken Cole, and Art Phillips for their comments and discussions about packrat middens. We also appreciate the help of the numerous additional collectors cited within the paper. Larry Arnold helped analyze the samples in the laboratory. Financial support was supplied by the National Science Foundation to P S Martin (DEB75-13944), T R Van Devender (DEB7619784) and to $\mathrm{P} E$ Damon and A Long (EAR74-13362 AO2). The ${ }^{14} \mathrm{C}$ laboratory is also supported in part by the State of Arizona. University of Arizona, Department of Geosciences Contribution No. 809.

\section{SAMPLE DESCRIPTIONS}

\section{Colorado Plateau}

\section{Wide Rock Series}

Two samples from Wide Rock, WdR, an isolated butte in Canyon de Chelly at $2100 \mathrm{~m}$ alt $\left(36^{\circ} 05^{\prime} \mathrm{N}, 109^{\circ} 20^{\prime} \mathrm{W}\right)$, Navajo Co, Arizona. The indurated midden ( 15 by 1 by $0.76 \mathrm{~m}$ ) contains 4 levels (Schmutz et al, 1976). Coll by Arthur Dennis; subm by Jeff Zauderer. 
General Comment: with such a large midden of many levels, the 2 dates should be considered exploratory in nature.

A-1536. WdR Level 2, $45 \mathrm{~cm}$ $1930 \pm 80$ Juniperus osteosperma twigs.

\section{A-1537. WdR Level 4, $76 \mathrm{~cm}$}

$6210 \pm 90$

Neotoma dung.

\section{Grand Canyon}

Middens were recovered from numerous canyons within the Grand Canyon. Canyons are referenced to their Colorado $\mathrm{R}$ mile location, following the conventional usage of miles (Hamblin \& Rigby, 1968).

\section{Horseshoe Mesa}

One sample from Crystal Forest Cave, Horseshoe Mesa, S of Colorado R Mile 77, Grand Canyon ( $\left.36^{\circ} 01^{\prime} \mathrm{N}, 111^{\circ} 58^{\prime} \mathrm{W}\right)$, Coconino Co, Arizona. Coll by KLC 1976; subm by KLC and PSM.

\section{A-1805. Horseshoe Mesa 7a, 1424m alt \\ $13,540 \pm 170$

Juniperus sp twigs.

\section{Hance Canyon series}

Two samples from Bida Cave, Hance Canyon, $S$ of Colorado R Mile 77.5, Grand Canyon ( $36^{\circ} 00^{\prime} \mathrm{N}, 111^{\circ} 57^{\prime} \mathrm{W}$ ), Coconino Co, Arizona. Coll by KLC 1976; subm by KLC and PSM.

\section{A-1789. Bida Cave 2, 1424m alt}

$$
14,200 \pm 470
$$

Pseudotsuga menziesii and Abies concolor needles.

\section{A-1790. Bida Cave 2c, 1424m alt}

Plant and midden debris.

\section{Shinumo Creek series}

Two samples from $\mathrm{N}$ of Colorado $\mathrm{R}$ Mile 107.6, Grand Canyon $\left(36^{\circ} 14^{\prime} \mathrm{N}, 112^{\circ} 21^{\prime} \mathrm{W}\right)$, Cononino Co, Arizona (Van Devender \& Mead, 1976; Cole and Van Devender, 1976; Van Devender et al, 1977). Midden not in Shinumo Creek but closer to Bass Rapids. Coll by PSM, JIM and J E King 1969 and 1976; subm by PSM, JIM, and TRV.

General Comment: Shinumo Creek samples represent 2 different samples from the same large midden.

\section{A-1321. SC 1, 730m alt}

$13,660 \pm 160$

Juniperus sp twigs.

$$
\delta^{13} \mathrm{C}=-22.5 \%
$$

A-1444. SC 1, 730m alt

$12,070 \pm 600$

Opuntia cf whipplei spines. 


\section{Peach Springs Wash series}

Sample from Peach Springs Wash (PSW), $11 \mathrm{~km}$ S of Diamond Creek at confluence with Colorado R Mile 225.6, Grand Canyon, Mohave Co, Arizona.

\section{A-1454. PSW 1, 885m alt}

$12,040 \pm 250$

Juniperus sp twigs ( $35^{\circ} 47^{\prime} \mathrm{N}, 113^{\circ} 30^{\prime} \mathrm{W}$ ) (Van Devender \& Mead, 1976; Cole and Van Devender, 1976; Van Devender et al, 1977). Coll by TRV and JIM 1973; subm by TRV and JIM.

\section{A-1718. Cave of the Early Morning Light, $1300 m$ alt}

$$
\begin{array}{r}
\mathbf{1 6 , 5 8 0} \pm \mathbf{4 6 0} \\
\delta^{13} C=-21.5 \% \text { o }
\end{array}
$$

Juniperus sp twigs $\left(35^{\circ} 42^{\prime} 45^{\prime \prime} \mathrm{N}, 113^{\circ} 23^{\prime} 20^{\prime \prime} \mathrm{W}\right)$. Coll by Don Davis 1976; subm by TRV and PSM.

\section{Vulture Canyon series}

Samples from Vulture Canyon (VC), S of Colorado R Mile 274.5, Grand Canyon ( $36^{\circ} 06^{\prime} \mathrm{N}, 113^{\circ} 56^{\prime} \mathrm{W}$ ), Mohave Co, Arizona. Samples are from numerous isolated rock crevice middens throughout predominantly limestone canyon and from Vulture Cave (Phillips \& Van Devender, 1974; Cole \& Van Devender, 1976; Phillips, 1977; Van Devender et al, 1977; Mead \& Phillips, ms in prep). Coll by AMP, JIM, TRV, and PSM 1972 to 1976; subm by AMP, JIM, TRV, and PSM.

\section{A-1316. VC 1, 540m alt}

$$
12,770 \pm 440
$$$$
\delta^{13} C=-22.3 \%
$$

Juniperus sp twigs. Comment: VC $1=$ Columbine Falls 1 .

\section{A-1564. VC 8 Vulture Cave, 645m alt $\quad 13,820 \pm 220$}

Juniperus sp twigs. Comment: youngest dated midden in cave. Sample dates only a single small unit within extremely complex, stratified midden. Date not representative of entire midden.

A-1566. VC 4, 495m alt

Juniperus sp seeds and twigs.

A-1567. VC 2b, 460m alt

Juniperus sp twigs.

\section{A-1568. VC 12, 675m alt}

Fraxinus anomala seeds and twigs.

A-1579. VC 5, 620m alt

Juniperus sp twigs. $\mathbf{1 0 , 6 1 0} \pm \mathbf{3 2 0}$
$\delta^{13} \mathrm{C}=-19.8 \% 0$

$$
10,250 \pm 290
$$

$\delta^{13} \mathrm{C}=-22.4 \%$ o

$$
8540 \pm 180
$$$$
\delta^{13} C=-21.8 \% \text { o }
$$

$$
13,400 \pm 390
$$

$\delta^{13} \mathrm{C}=-22.0 \%$ 
A-1587. VC 14, 645m alt

$11,870 \pm 190$

$\delta^{13} \mathrm{C}=-20.7 \%$

Juniperus sp seeds and twigs.

A-1603. VC 6 Vulture Cave, 645m alt

$17,610 \pm 290$

$\delta^{13} \mathrm{C}=-22.0 \%$

Juniperus sp twigs.

A-1604. VC 7 Vulture Cave, 645m alt

$22,720 \pm 610$

Juniperus sp twigs.

A-1605. VC 9 Vulture Cave, 645m alt

$29,810 \pm 1980$

$\delta^{13} \mathrm{C}=-21.8 \%$

Juniperus sp twigs. Comment: oldest dated midden from cave.

A-1606. VC 10 Vulture Cave, 645m alt

$19,050 \pm 390$

$\delta^{13} C=-21.8 \%$

Juniperus sp twigs.

A-1607. VC 13 Vulture Cave, 645m alt

$15,130 \pm 210$

$\delta^{13} C=-23.0 \%$

Juniperus sp twigs. Comment: unindurated floor midden covered by ca $5 \mathrm{~cm}$ eolian silt.

\section{A-1608. VC 15 Vulture Cave, 645m alt}

$15,260 \pm 270$

$\delta^{13} \mathrm{C}=-23.0 \%$

Juniperus sp twigs. Comment: apparent indurated extension of unindurated midden (see A-1607).

\section{Rampart Cave Series}

Samples from Rampart Cave (RG), S of Colorado R Mile 274.5, $535 \mathrm{~m}$ alt, Grand Canyon ( $\left.36^{\circ} 06^{\prime} \mathrm{N}, 113^{\circ} 56^{\prime} \mathrm{W}\right)$, Mohave Co, Arizona. Cave contains $145 \mathrm{~cm}$ stratified sloth dung and unindurated packrat middens (Rat Layer). Indurated middens occur along cave wall and in excavated pits (Martin et al, 1961; Long \& Martin, 1974; Long et al, 1974; Phillips \& Van Devender, 1974; Cole \& Van Devender, 1976; Van Devender et al, 1977; Van Devender, 1977). Coll by AMP, TVD, PSM, and AL 1972 to 1976; subm by AMP, TRV, and PSM.

\section{A-1208. Rat Layer $71 \mathrm{~cm}$}

$16,700 \pm 900$

$\delta^{13} \mathrm{C}=-8.5 \%$

Neotoma dung from Ray Layer. Comment: date is minimum for layer; unindurated.

A-1209. Rat Layer $96 \mathrm{~cm}$

$23,540 \pm 460$

Neotoma dung at base of layer. Comment: date is maximum for layer; unindurated. 
A-1325. RC St 45

$13,170 \pm 310$

Juniperus sp seeds and twigs.

A-1350. RC Pit B Original

$12,600 \pm 260$

$\delta^{13} \mathrm{C}=-22.7 \%$ 。

Juniperus sp twigs.

A-1356. RC Rat Layer

$18,890 \pm 500$

Fraxinus anomala seeds and twigs. Comment: unindurated.

A-1421. RC Roof Crack

$13,510 \pm 190$

Juniperus sp twigs.

A-1450. RC St 50

$9770 \pm 160$

Juniperus sp twigs.

$\delta^{13} C=-22.0 \%$

A-1451. RC St 50

$9520 \pm 400$

Fraxinus anomala twigs and seeds.

\section{A-1452. RC St 50}

$9520 \pm 330$

Single Agave utahensis leaf fragment.

$$
\delta^{13} \mathrm{C}=-11.9 \%
$$

A-1453. RC St 50

$11,140 \pm 250$

Nothrotheriops shastense dung. Comment: A-1450 and -1453 are on different midden constituents from same unit sample. Three are within $1 \sigma$, whereas A-1453 represents incorporation of older material into a younger midden.

\section{A-1535. RC St 35}

$12,230 \pm 350$

Juniperus sp and Fraxinus anomala seeds and twigs.

\section{A-1569. RC Pit B Front}

$16,330 \pm 270$

Juniperus sp twigs.

$\delta^{13} \mathrm{C}=-21.4 \%$ o

\section{A-1570. RC Rat Layer}

$14,810 \pm 220$

Juniperus sp single twig.

$$
\delta^{13} C=-20.6 \%
$$

\section{A-1791. RC Rat Layer}

$26,300 \pm 760$

$\delta^{1 s} C=-21.2 \%$ 1974.

Equus sp (small) hoof. Subm by TRV and KLC 1977; coll by AMP 


\section{Needle-Eye Canyon}

One sample has been taken from Needle-Eye Canyon (NC), $\mathrm{N}$ of Colorado R Mile 276.5, Grand Canyon ( $36^{\circ} 06^{\prime} \mathrm{N}, 113^{\circ} 56^{\prime} \mathrm{W}$ ), Mohave Co, Arizona (Phillips, 1977; Van Devender et al, 1977; Van Devender, 1977). Coll by AMP, TRV, and PSM 1973; subm by AMP, PSM, and TRV.

A-1618. NC 1, 550m alt

$9720 \pm 260$

Juniperus sp twigs.

$$
\delta^{13} C=-23.0 \%
$$

\section{Window Rock Canyon series}

Window Rock Canyon (WR), N of Colorado R Mile 275.2, Grand Canyon ( $36^{\circ} 06^{\prime} \mathrm{N}, 113^{\circ} 56^{\prime} \mathrm{W}$ ), Mohave Co, Arizona (Cole \& Van Devender, 1976; Phillips, 1977; Van Devender et al, 1977). Coll by TRV and PSM 1973; subm by TRV and PSM.

General Comment: assoc with Agave, Yucca, and Vitis.

A-1314. WR 1 level 1h, 465m alt

Juniperus sp twigs.

\section{A-1352. WR 2c, $465 m$ alt}

Juniperus sp twigs.

$$
\begin{array}{r}
11,310 \pm \mathbf{3 8 0} \\
\delta^{1 s} C=-23.5 \% 0
\end{array}
$$

$$
10,250 \pm 220
$$$$
\delta^{13} C=-23.1 \%
$$

\section{Desert Almond Canyon series}

Formerly Emergy Falls Canyon (Phillips \& Van Devender, 1974; Cole \& Van Devender, 1976; Van Devender et al, 1977), Desert Almond Canyon (DA) is $\mathrm{N}$ of Colorado R Mile 276.5, Grand Canyon $\left(36^{\circ} 06^{\prime} \mathrm{N}, 113^{\circ} 56^{\prime}\right.$ W), Mohave Co, Arizona. Samples are from isolated middens in limestone shelters within canyon (Phillips, 1977; Van Devender, 1977). Coll by AMP, TRV, and PSM 1973; subm by AMP, PSM, and TRV.

A-1380. DA 1a,b, 490m alt

Nolina microcarpa leaves.

A-1422. DA 3b, 520m alt

Juniperus sp twigs.

A-1423. DA 4, 585m alt Juniperus sp twigs.

A-1426. DA 2a, 490m alt
$10,100 \pm 200$ $\delta^{13} C=-24.6 \%$

$11,190 \pm 150$ $\delta^{13} C=-22.6 \%$

$$
11,990 \pm 490
$$

$10,930 \pm 460$ $\delta^{13} C=-23.4 \%$

Nolina microcarpa, Prunus fasciculata, and Fraxinus anomala leaves and twigs. 
A-1427. DA 6a,b, 570m alt

$$
\begin{array}{r}
10,910 \pm 450 \\
\delta^{13} C=-21.6 \% 0
\end{array}
$$

Nolina microcarpa and Fraxinus anomala leaves and twigs.

\section{A-1428. DA $7 \mathrm{~b}, 560 \mathrm{~m}$ alt}

$9650 \pm 360$

Juniperus sp twigs.

A-1456. DA 5a,b, 565m alt

$10,450 \pm 420$ twigs.

Mortonia scarbella, Juniperus sp, and Fraxinus anomala leaves and

A-1469. DA 7a, 560m alt

$8560 \pm 260$

Juniperus sp twigs.

A-1547. DA 8, 575m alt

$8850 \pm 150$

Nolina microcarpa leaves.

\section{A-1720. DA 10a, 635m alt}

$12,650 \pm 380$

Juniperus sp twigs.

\section{Muav Gate Canyon}

Muav Gate Canyon (MG) is $\mathbf{N}$ of Colorado $\mathrm{R}$ Mile 275.2, Grand Canyon $\left(36^{\circ} 06^{\prime} \mathrm{N}, 113^{\circ} 56^{\prime} \mathrm{W}\right.$ ), Mohave Co, Arizona (Cole \& Van Devender, 1976; Phillips, 1977; Van Devender et al, 1977). Coll by TRV and PSM 1973; subm by PSM and TRV.

A-1455. MG 1, 440m alt

$12,430 \pm 550$

Juniperus sp twigs.

\section{Iceberg Canyon}

One sample from Iceberg Canyon (IC) S of Colorado R Mile 287.2 $\left(36^{\circ} 11^{\prime} \mathrm{N}, 114^{\circ} 03^{\prime} \mathrm{W}\right)$, Mohave 'Co, Arizona (Phillips, 1977). Coll by TRV and WGS 1973; subm by AMP, TRV, and PSM.
A-1322.
IC 1, 425m alt
$11,010 \pm 400$
$\delta^{18} \mathrm{C}=-21.9 \%$

Juniperus sp twigs.

\section{Mohave Desert}

\section{Sheep Range series}

Samples are from an elevational transect of the Sheep Range (36 $29^{\prime} \mathrm{N}, 115^{\circ} 15^{\prime} \mathrm{W}$ ), Clark Co, Nevada (Spaulding, 1977). Coll by WGS 1972 to 1975 ; subm by WGS and PSM.

A-1379. Long Canyon Saddle 1, 1700m alt $\quad 30,400 \pm 1500$ Juniperus osteosperma twigs. Comment: Long Canyon Saddle $1=$ Fleharty Canyon Saddle 1. 
A-1531. Canyon Two top layer, 1740m alt $\quad 3310 \pm 100$

Juniperus osteosperma twigs and seeds. Comment: assoc with Yucca brevifolia and Opuntia basilaris.

A-1532. Canyon Two bottom layer, 1740m alt $1990 \pm 70$

Juniperus osteosperma twigs. Comment: assoc with Yucca brevifolia and Opuntia basilaris.

A-1642. Spires 2, 2030m alt

$>\mathbf{2 6 , 0 0 0}$
$\delta^{13} C=-21.5 \%$

Pinus longaeva and $P$ flexilis needles.

A-1643. Tire Wash 1, 1750m alt

$$
\begin{array}{r}
>\mathbf{3 4 , 0 0 0}, 0 \\
\delta^{1 s} C=-21.4 \% \text { o }
\end{array}
$$

Juniperus osteosperma twigs.

A-1656. Tire Wash 2, 1790m alt

$25,000 \pm 710$

Juniperus osteosperma twigs.

$\delta^{13} C=-22.0 \%$

A-1665. Spires 1, 2060m alt

$3460 \pm 150$

Yucca baccata leaf.

$\delta^{13} C=-11.7 \%$

A-1669. South Crest Level 3, 1970m alt

$\mathbf{2 5 , 2 0 0} \pm \mathbf{9 0 0}$

Juniperus osteosperma twigs.

A-1670. Spires 3, 2000m alt

$9540 \pm 130$

Juniperus osteosperma twigs.

$\delta^{13} C=-22.6 \%$

A-1725. Willow Wash 1a, 1560m alt

$22,420 \pm 720$

Juniperus osteosperma seeds and twigs.

$\delta^{13} C=-21.3 \%$

A-1740. Willow Wash 5, 1570m alt

$\begin{aligned} & >\mathbf{3 1 , 0 0 0} \\ \delta^{13} C= & -21.7 \%\end{aligned}$

Juniperus osteosperma seeds and twigs.

\section{Potosi Mountain}

One sample from a limestone cave at $\mathrm{N}$ end of Potosi $\mathrm{Mt}$ in the Spring Range, (PM) (36 $\left.00^{\prime} \mathrm{N}, 115^{\circ} 30^{\prime} \mathrm{W}\right)$, Clark Co, Nevada. Coll by RST and JIM 1976; subm by JIM, RST, and PSM.

A-1778. PM 2c $c_{2}, 1830 m$ alt

$14,450 \pm 250$

Abies concolor needles. 


\section{Newberry Mountains}

Two samples from Newberry Mts $\left(35^{\circ} 16^{\prime} \mathrm{N}, 114^{\circ} 37^{\prime} \mathrm{W}\right)$, Clark Co, Nevada (Leskinen, 1975). Coll by P H Leskinen 1970; subm by P H Leskinen.

A-1017. Site 3, 850m alt

$9500 \pm 240$

Quercus dunnii acorns. Comment (PHL): species is not known to be living now in Nevada.
A-1136. Site 4, 850m alt
$15,000 \pm 1600$
$\delta^{13} C=-23.1 \%$

Juniperus osteosperma twigs. Comment (PHL): species no longer grows in Newberry Mts.

\section{Escondito Canyon}

\section{Sonoran Desert}

A modern midden from Escondito Canyon $\left(29^{\circ} 40^{\prime} \mathrm{N}, 112^{\circ} 31^{\prime} \mathrm{W}\right)$, Sonora, Mexico. Coll by PSM and Mary Kay O'Rourke 1975; subm by PSM and O'Rourke.
A-1674. Escondito Canyon 1, 70m alt

$$
\delta^{1 s} C \stackrel{320 \pm 65}{=}-21.9 \%
$$

Jatropha sp seeds.

\section{Tucson Mountains series}

Three samples from isolated middens in rock crevices in Tucson Mts (TM) $\left(32^{\circ} 19^{\prime} \mathrm{N}, 111^{\circ} 12^{\prime} \mathrm{W}\right)$ Pima Co, Arizona (Van Devender, 1973; Petit, 1974; Cole \& Van Devender, 1976; King \& Van Devender, 1977; Van Devender \& Mead, in press).

\section{A-994. TM 3, 700m alt}

$$
\begin{array}{r}
21,000 \pm 700 \\
\delta^{13} C=-19.1 \% 0
\end{array}
$$

Neotoma dung. Coll by P J Mehringer and P E Damon 1967; subm by Mehringer and Damon. Comment: TM $3=$ Contzen Pass 1 .
A-1195. TM 1, 890m alt
$12,430 \pm 400$ $\delta^{13} \mathrm{C}=-22.6 \%$

Juniperus sp twigs. Coll by TRV and K B Moodie 1970; subm by PSM and TRV.

A-1235. TM 2, 700m alt $2720 \pm 100$

Neotoma dung and plant debris. Coll by TRV and J E King 1971; subm by PSM, TRV, and King.

\section{Silver Bell Mountains series}

Four samples from 3 middens on Wolcott Peak (WP) in Silver Bell Mts $\left(32^{\circ} 27^{\prime} \mathrm{N}, 111^{\circ} 28^{\prime} \mathrm{W}\right)$ Pima Co, Arizona. All middens from $860 \mathrm{~m}$ alt (Van Devender, 1973; Cole \& Van Devender, 1976; Van Devender \& Mead, in press).

General Comment: Juniperus presently does not grow in these mts. 
A-1216. WP 2

Juniperus sp twigs and plant debris. Coll by TRV, K B Moodie 1971; subm by Moodie. Comment: WP 2 = Sawtooth 2.

A-1236. WP 4

$$
\begin{array}{r}
\mathbf{5 3 5 0} \pm \mathbf{1 0 0} \\
\delta^{13} C=-20.7 \% \text { o }
\end{array}
$$

Plant debris. Coll by TRV and K B Moodie 1971; subm by TRV.

\section{A-1286. WP 2}

$$
\begin{array}{r}
14,550 \pm 800 \\
\delta^{13} C=-19.6 \% 0
\end{array}
$$

Juniperus osteosperma twigs. Coll by PSM, TRV, and $\mathrm{K}$ B Moodie 1971; subm by TRV. Comment: this sample and A-1216 indicate that some intermixing from 2 different midden units has occurred.

\section{A-1287. WP 5}

$$
\begin{array}{r}
12,130 \pm \mathbf{5 0 0} \\
\delta^{13} C=-21.8 \% \text { o }
\end{array}
$$

Juniperus osteosperma twigs. Coll by PSM, TRV, and $\mathrm{K}$ B Moodie 1971; subm by TRV.

\section{Ajo Mountain series}

A single multilayered midden from a rock shelter on Montezuma's Head (MH) in the Ajo Mts $\left(32^{\circ} 07^{\prime} \mathrm{N}, 112^{\circ} 42^{\prime} \mathrm{W}\right)$ at $975 \mathrm{~m}$ alt, Pima Co, Arizona. Coll by TRV and PSM 1976; subm by TRV and PSM.

\section{A-1695. MH la}

$$
\mathbf{2 0 , 4 9 0} \pm \mathbf{5 1 0}
$$

Juniperus sp twigs.

$$
\delta^{13} C=-22.4 \%
$$

\section{A-1696. MH $1 \mathrm{~b}$}

$$
21,840 \pm 650
$$

Pinus monophylla needles.

$$
\delta^{13} C=-21.4 \% \text { o }
$$

\section{A-1697. MH Ic}

$$
17,830 \pm 870
$$

Pinus monophylla needles and Juniperus sp twigs.

$$
\delta^{13} C=-20.9 \%
$$

\section{A-1698. MH 1d}

$13,500 \pm 390$

Juniperus sp twigs.

$$
\delta^{13} C=-22.9 \% \text { o }
$$

\section{Wellton Hills series}

This series of Holocene samples was taken from small rock crevices in the Wellton Hills (WH) $\left(32^{\circ} 36^{\prime} \mathrm{N}, 114^{\circ} 07^{\prime} \mathrm{W}\right)$ Yuma Co, Arizona (Van Devender, 1973; Cole \& Van Devender, 1976; Van Devender \& Mead, in press). Coll by TRV 1973; subm by TRV and PSM.

\section{A-1364. WH 5a, 175m alt}

$$
8150 \pm 260
$$

Ephedra nevadensis twigs. 
A-1365. WH 5a, 175m alt

Larrea divaricata twigs and leaves. Comment: surface contamination by local species.

A-1399. WH 2b top, 160m alt

Ephedra nevadensis twigs.

A-1400. WH 2b top, 160m alt

$7950 \pm 370$

Larrea divaricata twigs and leaves. Comment: surface contamination by local species.

A-1406. WH 1, 160m alt

$10,750 \pm 400$

Ephedra nevadensis twigs.

A-1407. WH 1, 160m alt

$\mathbf{1 0 , 5 8 0} \pm \mathbf{5 5 0}$ Larrea.

Larrea divaricata twigs and leaves. Comment (TRV): oldest date on

\section{Kofa Mountain series}

Three midden samples from rock crevices in Kofa Mts, Yuma Co, Arizona. Burro Canyon at $\left(33^{\circ} 24^{\prime} \mathrm{N}, 114^{\circ} 01^{\prime} \mathrm{W}\right), 860 \mathrm{~m}$ alt. Brass Cap Point at $\left(33^{\circ} 26^{\prime} \mathrm{N}, 114^{\circ} 05^{\prime} \mathrm{W}\right), 550 \mathrm{~m}$ alt (Van Devender, 1973; Lanner \& Van Devender, 1974; King \& Van Devender, 1977; Van Devender \& Mead, in press).

\section{A-1315. Burro Canyon 1, Level $1 \quad 14,400 \pm 330$}

$\delta^{1 s} C=-21.6 \%$

Juniperus osteosperma twigs. Coll by A Gottesfeld 1971; subm by PSM and TRV.
A-1357. Burro Canyon 1, Level 6
$13,400 \pm 250$
$\delta^{13} C=-21.6 \%$

Juniperus sp twigs. Coll by TRV 1972; subm by PSM and TRV.

\section{A-1328. Brass Cap Point 1 \\ $11,450 \pm 400$ \\ $\delta^{13} C=-17.6 \%$}

Yucca brevifolia leaves. Coll by TRV 1972; subm by PSM and TRV.

New Water Mountains series

This series of midden samples from rock crevices in New Water Mts (NW) (33 $\left.36^{\prime} \mathrm{N}, 113^{\circ} 55^{\prime} \mathrm{W}\right)$ Yuma Co, Arizona (Van Devender, 1973; Lanner \& Van Devender, 1974; Petit, 1974; Cole \& Van Devender, 1976; King \& Van Devender, 1977; Van Devender, 1977; Van Devender \& Mead, in press).

\section{A-1284. NW 2, 615m alt}

$$
\begin{array}{r}
\mathbf{7 8 7 0} \pm \mathbf{7 5 0} \\
\delta^{13} \mathrm{C}=-12.5 \%
\end{array}
$$

Juniperus osteosperma seeds and twigs. Coll by W VanAsdall, L J Reynolds, and R W Sherwin 1971; subm by TRV. 


\section{A-1285. NW 4, 615m alt}

$\mathbf{1 0 , 8 8 0} \pm 900$

$\delta^{13} C=-20.5 \%$

Juniperus osteosperma twigs. Coll by W VanAsdall, L J Reynolds, and R W Sherwin 1971; subm by PSM and TRV.

\section{A-1295. NW 7, 605m alt}

$$
\mathbf{1 1 , 0 0 0} \pm \mathbf{5 1 0}
$$

$\delta^{13} C=-20.4 \%$

Juniperus sp twigs. Coll by J E King and TRV 1971; subm by PSM and TRV.

\section{A-1296. NW 7, 605m alt}

$$
\begin{array}{r}
\mathbf{2 7 1 0} \pm \mathbf{2 8 0} \\
\delta^{13} \mathrm{C}=-17.0 \% \text { o }
\end{array}
$$

Larrea divaricata twigs and leaves. Coll by J King and TRV 1971; subm by PSM and TRV.

\section{$\begin{array}{ll}\text { A-1353. NW 2, 615m alt } & 11,060 \pm 390\end{array}$}

Juniperus sp whole seeds and twigs. Coll by $\mathrm{W}$ VanAsdall and L J Reynolds 1971; subm by PSM and TRV.
A-1445. NW 2, 615m alt
$12,090 \pm 570$
$\delta^{13} C=-21.6 \%$

Quercus turbinella leaves. Coll by W VanAsdall and L J Reynolds 1971; subm by PSM and TRV.

\section{Artillery Mountain series}

Three middens were sampled from 2 rock shelters in Artillery Mts (A), Mohave Co, Arizona. A 1 and 3 at $\left(34^{\circ} 20^{\prime} \mathrm{N}, 113^{\circ} 35^{\prime} \mathrm{W}\right), 615 \mathrm{~m}$ alt. A 2 at $\left(34^{\circ} 20^{\prime} \mathrm{N}, 113^{\circ} 38^{\prime} \mathrm{W}\right), 725 \mathrm{~m}$ alt (Van Devender \& King, 1971; Van Devender, 1973; Lanner \& Van Devender, 1974; King \& Van Devender, 1977; Van Devender \& Mead, in press). Coll by TRV, PSM, K B Moodie, and J E King 1969 and 1970; subm by TRV and PSM.
A-1099. A 1
$10,250 \pm 200$
Juniperus sp twigs.

A-1100. A 3

$>\mathbf{3 0 , 0 0 0}$

Juniperus osteosperma twigs.

A-1101. A 2

$18,320 \pm 400$

Juniperus osteosperma and J. monosperma twigs.

A-1103. A 2

$$
\begin{array}{r}
\mathbf{1 8 4 0} \pm \mathbf{2 0 0} \\
\delta^{1 s} C=-13.0 \% 0
\end{array}
$$

Pinus monophylla needles. Comment: 1st run produced insufficient gas volume for a count. A 2nd run produced a very young age on very little gas and may be in error.

\section{Whipple Mountains series}

These middens are from rock crevices and a small cave in the Whipple Mts area of San Bernardino Co, California. The Falling Arches 
(FA) and Tunnel Ridge (TR) samples are at ( $\left.34^{\circ} 13^{\prime} \mathrm{N}, 114^{\circ} 22^{\prime} \mathrm{W}\right)$. The Redtail Peak (RP) samples are at $\left(34^{\circ} 16^{\prime} \mathrm{N}, 114^{\circ} 25^{\prime} \mathrm{W}\right)$ and the Whipple Mt proper (WM) samples are from $\left(34^{\circ} 14^{\prime} \mathrm{N}, 114^{\circ} 2^{\prime} \mathrm{W}\right.$ ) (Cole \& Van Devender, 1976; King \& Van Devender, 1977; Van Devender, 1977; Van Devender \& Mead, in press). Redtail Peak samples coll by TRV, JIM, and AMP 1974; subm by PSM and TRV.

A-1548. FA 1, 320m alt

$11,650 \pm 190$

Juniperus sp twigs. Coll by PSM, TRV, and Paul Johnson 1974; subm by PSM and TRV.

\section{A-1470. TR 2, 365m alt}

$$
\begin{array}{r}
\mathbf{1 0 , 3 3 0} \pm \mathbf{3 0 0} \\
\delta^{1 s} \mathrm{C}=-19.6 \%
\end{array}
$$
and Bull.

Juniperus sp twigs. Coll by AL and W B Bull 1973; subm by AL

\section{A-1550. TR 5, 365m alt}

$12,670 \pm 260$

Yucca brevifolia leaves. Coll by PSM, TRV, JIM, AMP and W B Bull 1974; subm by PSM and TRV.

\section{A-1582. TR 5a, 365m alt}

$$
12,330 \pm 350
$$$$
\delta^{13} \mathrm{C}=-21.0 \%
$$
PSM.

Juniperus sp twigs. Coll by TRV and AMP 1974; subm by TRV and

A-1580. RP 1, 520m alt

$8910 \pm 380$

$\delta^{13} C=-20.6 \%$

Juniperus californica twigs. Comment: assoc with Yucca brevifolia, Yucca whipplei, and Nolina bigelovii.

A-1616. RP 10a, 490m alt

Nolina bigelovii leaves.

\section{A-1620. RP 3, 510m alt}

Juniperus sp twigs.

\section{A-1621. RP 8, 495m alt}

Juniperus sp twigs.

A-1650. RP 11, 520m alt

Juniperus sp twigs.

A-1655. RP 6, 495m alt

Juniperus sp twigs.
$10,840 \pm 170$

$\delta^{13} C=-19.5 \%$

$10,030 \pm 160$

$\delta^{13} C=-20.0 \%$ o

$11,520 \pm 160$

$\delta^{13} C=-20.2 \%$ o

$13,810 \pm 270$

$\delta^{13} C=-20.5 \%$ 。

$9600 \pm 170$

$\delta^{13} C=-19.7 \%$ 
A-1661. RP 5, 510m alt

$10,880 \pm 180$ $\delta^{13} C=-19.3 \%$

Juniperus sp twigs.

A-1662. RP 5, 510m alt

$\mathbf{1 0 , 3 6 0} \pm \mathbf{3 5 0}$ $\delta^{13} C=-21.5 \%$

Midden debris with Nolina bigelovii, Juniperus sp, Amsinckia tessellata, Ephedra nevadensis, and Salvia mohavensis.

A-1663. RP 5, 510m alt

$9600 \pm 160$

Nolina bigelovii leaves. $\delta^{13} \mathrm{C}=-23.2 \%$

A-1664. RP 5, 510m alt

$10,540 \pm 140$

Neotoma dung. $\delta^{13} C=-22.2 \%$ o

A-1666. RP 5, 510m alt

$12,960 \pm 210$

Pinus monophylla wood. $\delta^{13} C=-21.3 \%$

A-1668. RP 1, Sample 2, 520m alt

$9160 \pm 170$

Juniperus sp twigs.

$\delta^{13} C=-20.2 \%$ o

A-1538. WM 2, 520m alt

$9980 \pm 180$

Juniperus sp twigs. Coll by TRV, PSM, JIM, AMP, and W B Bull 1974; subm by PSM and TRV. Comment: midden on cave floor. Assoc sp include Yucca baccata and Nolina bigelovii.

A-1551. WM 3, 515m alt

$9920 \pm 130$

Nolina bigelovii leaves. Coll by PSM, TRV, JIM, AMP, and W B Bull 1974; subm by PSM and TRV.

A-1615. WM 1, 520m alt

$10,430 \pm 170$ $\delta^{13} C=-22.4 \%$ o

Iuniperus sp twigs. Coll by TRV and PSM, 1974; subm by PSM and TRV.

Chihuahuan Desert

\section{A-1581. Shafter 1 b, $1310 m$ alt}

$15,670 \pm 230$ $\delta^{13} \mathrm{C}=-20.4 \%$

Juniperus monosperma twigs. Rock crevice midden in Livingston Hills, Chinati Mts $\left(29^{\circ} 47^{\prime} \mathrm{N}, 104^{\circ} 22^{\prime} \mathrm{W}\right)$ Presidio Co, Texas (Van Devender et al, in press). Coll by R D Worthington and E Freeman 1973; subm by PSM and TRV. 
A-1612. Quitman Mountain 1, 1430m alt

$10,910 \pm 170$

$\delta^{13} \mathrm{C}=-22.0 \%$

Juniperus cf monosperma twigs. Rock crevice midden in Quitman Mts ( $31^{\circ} 07^{\prime} \mathrm{N}, 105^{\circ} 23^{\prime} \mathrm{W}$ ) Hudspeth Co, Texas (Van Devender \& Wiseman, in press). Coll by TRV 1975; subm by PSM and TRV.

A-1623. Steeruwitz Hills 1 Unit P4, 1430m alt $18,060 \pm 1320$ $\delta^{31} C=-25.1 \%$

Juniperus sp twigs. Rock crevice midden in Sierra Diablo (31 $07^{\circ} \mathrm{N}$, $105^{\circ} 09^{\prime}$ W) Hudspeth Co, Texas. Coll by TRV and D R Frost 1975; subm by PSM and TRV.

\section{Guadalupe Mountains series}

Three middens from Guadalupe Mts $\left(31^{\circ} 54^{\prime} \mathrm{N}, 104^{\circ} 50^{\prime} \mathrm{W}\right)$ Culberson Co, Texas, were sampled from 3 limestone caves. One sample is from previously excavated Williams Cave. Coll by TRV, AMP, WGS, and JIM 1974; subm by PSM and TRV. The other 2 samples are from 2 sloth caves (Van Devender \& Everitt, 1977; Van Devender et al, 1977; Van Devender $e t a l$, in press). Coll by TRV and WGS 1974; subm by PSM and TRV.

General Comment: Picea dates document late Pleistocene occurrence of spruce which no longer grows in Texas (TRV).

A-1540. William's Cave 2, 1495m alt $\quad 12,040 \pm 210$

Juniperus sp twigs. Comment: assoc with Pinus edulis, Rhus, and Quercus.

A-1539. Cave-09, 2000m alt

$13,000 \pm 730$ midden.

Picea sp needles. Comment: needles from unindurated floor-fill

A-1549. Cave-08, 2000m alt

$13,060 \pm 280$

Picea sp needles.

\section{Rocky Arroyo series}

Rocky Arroyo (RA) $\left(32^{\circ} 27^{\prime} \mathrm{N}, 104^{\circ} 28^{\prime} \mathrm{W}\right)$ at $1130 \mathrm{~m}$ alt, Eddy Co, New Mexico. Coll by TRV 1975; subm by TRV and PSM.

\section{A-1648. RA 1}

Juniperus sp twigs.

$10,560 \pm 150$

\section{A-1657. RA 2}

Agave lecheguilla leaves.

$$
\delta^{31} C=-23.5 \%
$$

$$
\begin{array}{r}
\mathbf{2 9 4 0} \pm \mathbf{1 9 0} \\
\delta^{13} C=-11.0 \% 0
\end{array}
$$

\section{Hueco Mountains series}

This large $\mathrm{mt}$ mass has been sampled for packrat middens at numerous localities within the range. Hueco Mts Site $1\left(31^{\circ} 42^{\prime} \mathrm{N}, 105^{\circ} 58^{\prime} \mathrm{W}\right.$ ), at 1280m alt, Hudspeth Co, Texas (Van Devender, 1977; Van Devender \& Wiseman, in press). 
A-1613. Hueco Mountains 1a

$$
\begin{array}{r}
9370 \pm 130 \\
\delta^{13} C=-12.6 \%
\end{array}
$$

Yucca torrey leaves. Coll by TRV, BLE and R D Worthington 1975; subm by TRV.
A-1614. Hueco Mountains 1b
$8150 \pm 170$
$\delta^{13} C=-21.2 \%$

Juniperus sp twigs. Coll by R D Worthington 1975; subm TRV.

Hueco Tanks State Park (31 $\left.55^{\prime} \mathrm{N}, 106^{\circ} 02^{\prime} \mathrm{W}\right)$ at $1420 \mathrm{~m}$ alt, El Paso Co, Texas.

\section{A-1624. Hueco Tanks State Park 1}

$$
\begin{array}{r}
\mathbf{1 3}, \mathbf{5 0 0} \pm \mathbf{2 5 0} \\
\delta^{1 s} \mathrm{C}=-23.2 \% 0
\end{array}
$$

Juniperus sp twigs. Coll by D H Riskind 1974; subm by TRV.

\section{A-1647. Hueco Tanks State Park 2}

$$
\begin{array}{r}
\mathbf{9 3 8 0} \pm \mathbf{2 7 0} \\
\delta^{13} \mathrm{C}=-10.1 \% \mathrm{o}
\end{array}
$$

Opuntia phaeacantha and $O$ violacea seeds. Coll by TRV and BLE 1975; subm by TRV.

Navar Ranch (NR) ( $31^{\circ} 53^{\prime} \mathrm{N}, 102^{\circ} 09^{\prime}$ W) El Paso Co, Texas (Van Devender, 1977). Coll by TRV and BLE 1975; subm by TRV.

\section{A-1644. NR 1b, 1340m alt}

$$
>\mathbf{3 4 , 0 0 0}
$$

Juniperus sp twigs.

A-1645. NR 3 Pine B, 1370m alt

$$
\begin{array}{r}
16,240 \pm 430 \\
\delta^{13} C=-21.5 \% o
\end{array}
$$

Juniperus sp twigs.

A-1646. NR 5, 1330m alt

$$
11,360 \pm 330
$$

Quercus pungens (acorn) fragments.

$$
\delta^{13} C=-25.2 \%
$$

A-1649. NR 4c, $1370 \mathrm{~m}$ alt

$$
\begin{array}{r}
\mathbf{8 9 2 0} \pm \mathbf{3 7 0} \\
\delta^{13} C=-24.1 \% \text { o }
\end{array}
$$
midden.

Quercus pungens (acorn) fragments. Comment: unindurated floor

A-1651. NR 4c, 1370m alt

$$
11,410 \pm 220
$$

Opuntia imbricata stems.

$$
\delta^{13} C=-9.8 \%
$$

A-1652. NR 3a top, $1370 \mathrm{~m}$ alt

$10,750 \pm 600$

Quercus pungens acorn fragments. 
A-1719. NR 1b, 1340m alt

Gorpherus agassizi dung. Comment: dung incorporated in midden.

Picture Cave (PG) (31 $\left.53^{\prime} \mathrm{N}, 106^{\circ} 08^{\prime} \mathrm{W}\right)$ at $1430 \mathrm{~m}$ alt, El Paso Co, Texas. Coll by TRV, BLE, and Deborah Goldberg 1975; subm by TRV.

\section{A-1699. PC 1d}

Juniperus sp twigs.

\section{A-1706. PC 1a}

Opuntia phaeacantha and $O$ violacea seeds.

Tank Trap Wash (TTW) at $\left(31^{\circ} 53^{\prime} \mathrm{N}, 106^{\circ} 09^{\prime} \mathrm{W}\right) 1340 \mathrm{~m}$ alt, E1 Paso Co, Texas (Van Devender, 1977). Coll by TRV, BLE, and Deborah Goldberg 1975; subm by TRV.

\section{A-1707. TTW 1, Level 2b}

Cercocarpus breviflorus wood.

\section{A-1708. TTW 1, Level 4}

Juniperus sp wood.

\section{A-1709. TTW 1, Level 3}

Juniperus sp twigs.

\section{A-1710. TTW 1, Level 5}

Pinus edulis needles, seeds, and cone scales.

\section{A-1721. TTW 1, Level 2a}

Juniperus sp twigs.

\section{A-1722. TTW 2}

Juniperus sp twigs.

\section{A-1723. TTW 1, Level 1, top}

Juniperus sp twigs.

$$
\begin{array}{r}
12,030 \pm \mathbf{2 1 0} \\
\delta^{13} \mathrm{C}=-22.1 \% 0
\end{array}
$$

$$
\begin{array}{r}
1530 \pm \mathbf{1 2 0} \\
\delta^{13} C=-10.6 \% \text { o }
\end{array}
$$

$$
\begin{aligned}
&>\mathbf{3 4 , 0 0 0} \\
& \delta^{13} C=-23.0 \% \\
&>\mathbf{3 4 , 0 0 0} \\
& \delta^{13} C=-20.0 \% \\
&>\mathbf{3 4 , 0 0 0} \\
& \delta^{13} C=-21.8 \% \circ
\end{aligned}
$$

$19,670 \pm 1150$ $\delta^{13} C=-21.4 \%$ o

$\begin{aligned} & >\mathbf{3 3 , 0 0 0} \\ \delta^{13} C= & -22.3 \%\end{aligned}$

$$
21,200 \pm 990
$$$$
\delta^{13} C=-22.1 \%
$$

$\begin{aligned} & >\mathbf{3 5 , 0 0 0} \\ \delta^{13} C & =-22,8 \% 0\end{aligned}$

\section{Organ Mountains series}

Bishops Cap (BC) is a limestone hill at S end of Organ Mts $\left(32^{\circ} 11^{\prime}\right.$ $\mathrm{N}, 106^{\circ} 36^{\prime} \mathrm{W}$ ) at $1465 \mathrm{~m}$ alt Doña Ana Co, New Mexico (Van Devender \& Everitt, 1977; Van Devender et al, 1977). 
A-1571. BC 1

$10,650 \pm 170$

Juniperus sp seeds and twigs.

\section{A-1572. BC 2}

$10,780 \pm 240$

Juniperus sp seeds and twigs.

\section{A-1573. BC 3}

$10,260 \pm 250$

Juniperus sp seeds and twigs.

Shelter Cave in Bishops Cap was first excavated in 1930 and is now known to contain dried sloth dung. A midden sample was removed from a crevice within the cave $\left(32^{\circ} 11^{\prime} \mathrm{N}, 106^{\circ} 36^{\prime} \mathrm{W}\right)$ Doña Ana Co, New Mexico. Coll by California Inst Technol 1930; subm by PSM, TRV, and Los Angeles Co Mus.

\section{A-1729. Shelter Cave, $1400 \mathrm{~m}$ alt}

$11,850 \pm 380$

$\delta^{13} C=-21.6 \%$

Midden debris with Juniperus sp twigs and Opuntia imbricata. Comment: no cave provenience given for midden (LACM 1010, N-6-11), midden debris attached to Gopherus agassizi scute.

\section{REFFRENCES}

Cole, C J and Van Devender, T R, 1976, Surface structure of fossil and recent epidermal scales from North American lizards of the genus Sceloporus (Reptilia, Iguanidae): Am Mus Nat Hist Bull, v 156, p 453-513.

Hamblin, W K and Rigby, J K, 1968, Guidebook to the Colorado River Part 1: Brigham Young Univ geol ser, v 15, pt 5, 84 pp.

King, J E and Van Devender, 'I R, 1977, Pollen analysis of fossil packrat middens from the Sonoran Desert: Quaternary Research, v 8, p 191-204.

Lanner, R M and Van Devender, T R, 1974, Morphology of pinyon pine needles from fossil packrat middens in Arizona: Forest Sci, v 20, p 207-211.

Leskinen, P H, 1970, Late Pleistocene vegetation change in the Christmas Tree Pass area, Newberry Mountains, Nevada: MS thesis, Univ Arizona, $39 \mathrm{p}$.

1975, Occurrence of oaks in late Pleistocene vegetation in the Mojave Desert of Nevada: Madroño, v 23, p 234-235.

Long, A, Hansen, R M, and Martin, P S, 1974, Extinction of the Shasta ground sloth: Geol Soc America Bull, v 85, p 1843-1848.

Long, A and Martin, P S, 1974, Death of American ground sloths: Science, v 186 , p 638-640.

Martin, P S, Sables, B E, and Shutler, D, Jr, 1961, Rampart Cave coprolite and ecology of the Shasta ground sloth: Am Jour Sci, v 259, p 102-127.

Petit, M G, 1974, The racemization rate constant for protein-bound aspartic acid in woodrat middens: Quaternary Research, v 4, p 340-345.

Phillips, A M, III, 1977, Packrats, plants and the Pleistocene in the lower Grard Canyon: PhD dissert, Univ Arizona, $123 \mathrm{p}$.

Phillips, A M, III and Van Devender, T R, 1974, Pleistocene packrat middens from the lower Grand Canyon of Arizona: Arizona Acad Sci Jour, v 9, p 117-119.

Schmutz, E M, Dennis, A E, Harlan, A, Hendricks, D, and Zauderer, J, 1976, An ecological survey of Wide Rock Butte in Canyon de Chelly National Monument, Arizona: Arizona Acad Sci Jour, v 11, p 114-125.

Spaulding, W G, 1977, Late Quaternary vegetational change in the Sheep Range, southern Nevada: Arizona Acad Sci Jour, v 12, p 3-8.

Stones, R C and Hayward, C L, 1968, Natural history of the desert woodrat, Neoioma lepida: Am Midland Naturalist, v 80, p 458-476. 
Van Devender, T R, 1973, Late Pleistocene plants and animals of Sonoran Desert: A survey of ancient packrat middens in southwestern Arizona: PhD dissert, Univ Arizona, $179 \mathrm{p}$. p 189-192.

1977, Holocene woodlands in the southwestern deserts: Science, v 198

Van Devender, T R and Everitt, B L, 1977, The latest Pleistocene and Recent vegetation of the Bishop's Cap, south-central New Mexico: SW Naturalist, v 22, p. 337-352.

Van Devender, T R, Freeman, C E, and Worthington, R D, in press, Full glacial and recent vegetation of Livingston Hills, Presidio County, Texas: SW Naturalist, v 23 ,

Van Devender, $T$ R and King, J E, 1971, Late Pleistocene vegetational records in western Arizona: Arizona Acad Sci Jour, v 6, p 240-244.

Van Devender, T R and Mead, J I, 1976, Late Pleistocene and modern plant communities of Shinumo Creek and Peach Springs Wash, lower Grand Canyon, Arizona: Arizona Acad Sci Jour, v 11, p 16-22.

1978, in press, Earth Holocene and late Pleistocene amphibian and reptiles in Sonoran Desert packrat middens: Copea, no. 3

Van Devender, T R, Moodie, K B, and Harris, A H, 1976, The descrt tortoise (Gopherus agassizi) in the Pleistocene of the northern Chihuahuan Desert: Herpetologica, v $32, \mathrm{p} 298-304$

Van Devender, T R, Phillips, A M, III, and Mead, J I, 1977, Late Pleistocene reptiles and small mammals from the lower Grand Canyon of Arizona: SW Naturalist, v 22, p 49-66.

Van Devender, $T$ R, Spaulding, W G, and Phillips, A M, in press, Late Pleistocene plant communities in the Guadalupe Mountains, Culberson County, Texas, in Biological investigations in the Guadalupe Mountains National Park, Texas: Symposium volume, Natl Park Service Pub.

Van Devender, $T$ R and Wiseman, F M, in press, A preliminary chronology of bioenvironmental changes during the PaleoIndian period in the monsoonal Southwest, in Johnson, J (cd), Symposium volume on PaleoIndian Lifeways: West Texas Mus Assoc Mus Bull, Lubbock, Texas.

Wells, P V, 1976, Macrofossil analysis of wood rat (Neotoma) middens as a key to the Quaternary vegetational history of arid America: Quaternary Research, v 6, p 223-248. 